\title{
An unusual cause of syncope
}

\author{
Abhilash Koratala ${ }^{1}$. Vikrampal Bhatti ${ }^{1}$
}

Received: 12 October 2016/Accepted: 22 October 2016/Published online: 27 October 2016 (c) SIMI 2016

A 78-year-old woman with a history of hypertension, diabetes mellitus type 2 and peripheral neuropathy was brought to the emergency department (ED) after a syncopal episode. She had a similar episode 1 month prior when she was seen at a local hospital and her anti-hypertensive medications were discontinued for presumed orthostatic hypotension. Stroke was ruled out at that time, and there was no reported hypoglycemia. She also complained of frequent dizziness, feet and ankle swelling, and low grade fevers for the prior 2 months. She had no pets and denied recent travel.

On examination, she was ill-appearing and afebrile; blood pressure was 108/66 $\mathrm{mmHg}$ and pulse $101 \mathrm{bpm}$. She had scleral icterus, abdominal distention, a mildly tender abdomen and $2+$ bilateral pitting pedal edema. An EKG was normal. Laboratory tests showed normal electrolytes, renal function and blood glucose. Liver function tests were: serum albumin $2.4 \mathrm{~g} / \mathrm{dL}$ (3.5-5), aspartate transaminase $42 \mathrm{U} / \mathrm{L}$ (0-37), alanine transferase $28 \mathrm{U} / \mathrm{L}(0-41)$, alkaline phosphatase $610 \mathrm{U} / \mathrm{L}$ (35-129), total bilirubin $3.7 \mathrm{mg} / \mathrm{dL}(0-1)$, direct bilirubin $2.5 \mathrm{mg} / \mathrm{dL}(0-0.2)$, INR 1.2 and negative viral hepatitis panel. Review of a recent echocardiogram did not show any pericardial effusion or significant valvular lesions. A CT scan of the abdomen was obtained to further investigate the etiology of her icterus and abnormal liver function tests, which revealed a large cyst in the left hepatic lobe measuring $\sim 15 \times 14 \times 14 \mathrm{~cm}$, significantly compressing the inferior vena cava without any obvious thrombosis (Figs. 1, 2; arrows point to the compressed inferior vena cava). Drainage of the cyst revealed approximately $1.8 \mathrm{~L}$ of greenish, non-purulent fluid with WBC count of $16,950 / \mathrm{mm}^{3}, 82 \%$ neutrophils, no malignant cells and culture was negative. She was started on intravenous piperacillin-tazobactam. Her dizziness quickly improved.

Compression of the inferior vena cava results in venous pooling in the lower extremities leading to pedal edema. Also, due to the decreased preload, patients may experience dizziness or syncope. Tachycardia and orthostatic hypotension may be noted. Our patient had these signs and symptoms together with laboratory findings suggestive of piperacillin-tazobactam etiology. In the absence of other common causes of syncope in this elderly lady and prompt resolution of her symptoms with drainage of the cyst, we conclude that compression of the inferior vena cava by hepatic cyst was the cause of her syncope. Cases of inferior vena cava thrombosis and pulmonary embolism have been reported with large hepatic cysts, and clinicians need to be aware of these potential complications [1,2]. Our case

Abhilash Koratala

akoratsla@ufl.edu

1 Division of Nephrology, Hypertension and Renal

Transplantation, University of Florida,

P.O. Box 100224, Gainesville, FL 32610, USA 


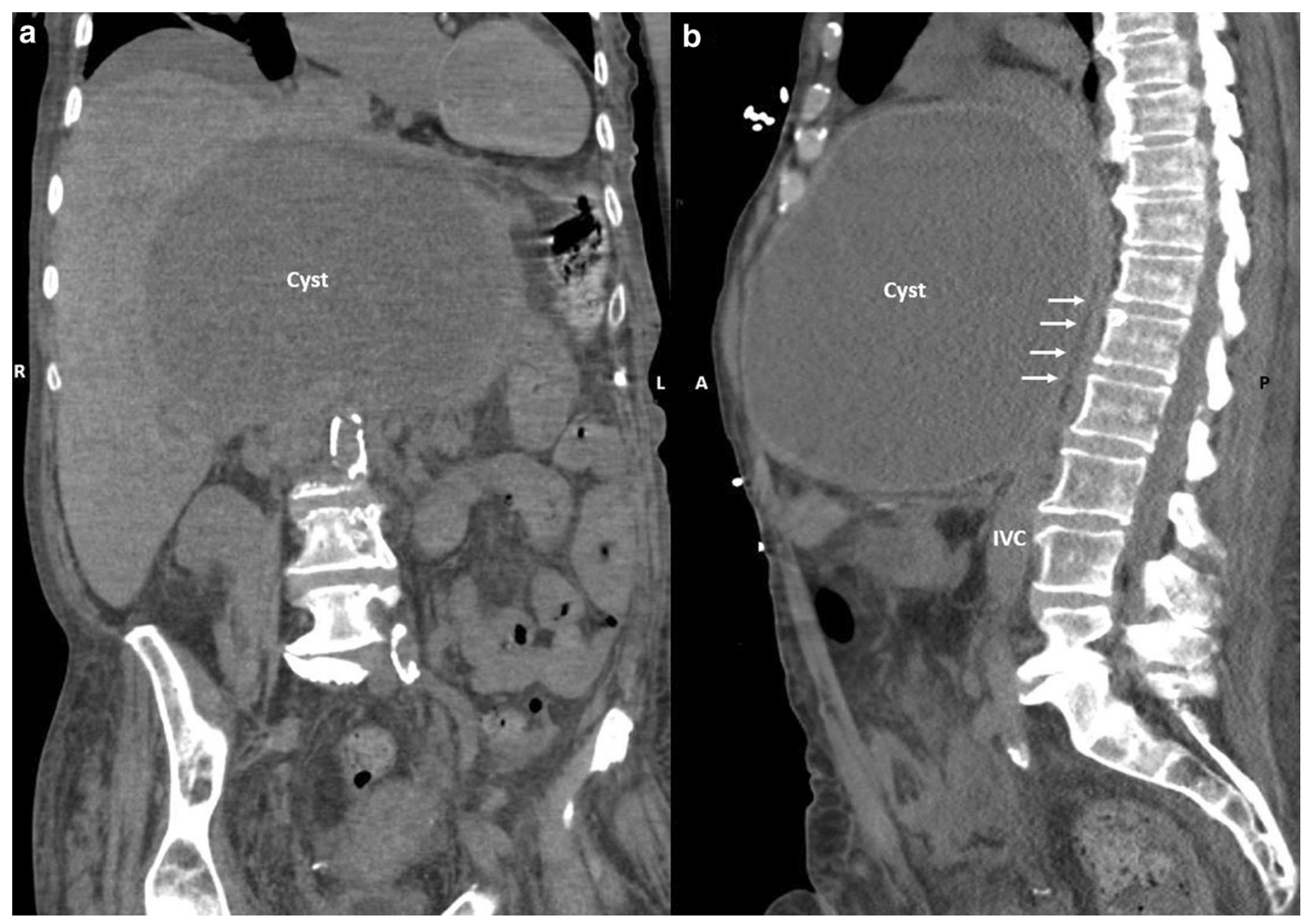

Fig. 1 a Coronal view of an abdominal CT scan showing a large cyst in the left hepatic lobe. b Sagittal view of an abdominal CT scan showing the hepatic cyst compressing the inferior vena cava (IVC). The arrows point to the IVC

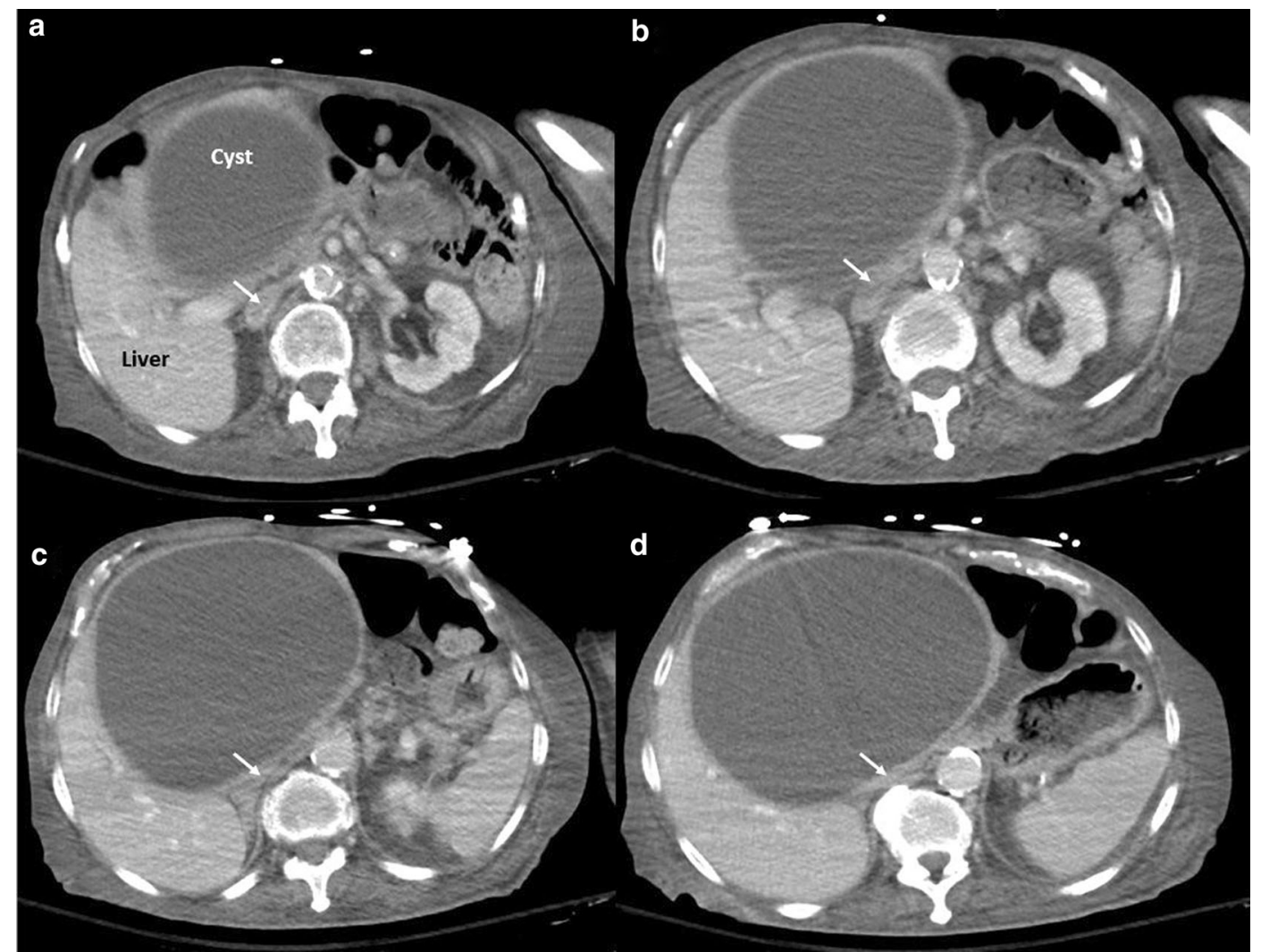

Fig. 2 a-d Transverse view of an abdominal CT scan showing progressive compression of the inferior vena cava (IVC) by the hepatic cyst. The arrow points to the IVC in each panel 
highlights the importance of thorough physical examination and timely investigations in recognizing a rare cause of a common symptom.

\section{Compliance with ethical standards}

Conflict of interest The authors declare that they have no conflict of interest.

Statement of human and animal rights All procedures followed were in accordance with the ethical standards of the responsible committee on human experimentation and with the Helsinki Declaration of 1975, as revised in 2013. No animals were used during the study.
Informed consent Informed consent was obtained from the patient for publication of this study.

\section{References}

1. Musielak MC, Singh R, Hartman E et al (2014) Simple hepatic cyst causing inferior vena cava thrombus. Int J Surg Case Rep 5(6):339-341

2. San Norberto EM, Fuente R, Taylor JH et al (2015) Endovascular management of inferior vena cava invasion by hepatic hydatid cyst. J Vasc Interv Radiol 26(1):144-146 